\title{
Riesz Transform on the Dual of the Laguerre Hypergroup
}

\author{
Mohamed Néjib Lazhari \\ Faculty of Sciences of Tunis, Department of Mathematics \\ Tunis 1060, Tunisia \\ E-mail: Mnejib.Lazhari@fst.rnu.tn \\ Taieb Ahmed (Corresponding author) \\ Faculty of Sciences of Tunis, Department of Mathematics \\ Tunis 1060 , Tunisia \\ E-mail: taiebahmed@yahoo.fr
}

Received: March 4, 2012 Accepted: March 31, 2012 Online Published: May 28, 2012

doi:10.5539/jmr.v4n3p50 URL: http://dx.doi.org/10.5539/jmr.v4n3p50

\begin{abstract}
In this paper, we define the Riesz transform on the dual of the Laguerre hypergroup associated with Plancherel measure and we give some properties for this transform. Also we investigate $L^{p}$-boundedness of this operator and under this definition, the higher order Riesz transform is given. Moreover we establish that the Riesz transform can be extended as a principal value singular integral operator and it is a multiplier operator under the Fourier transform.
\end{abstract}

Keywords: Laguerre hypergroup, Fourier-Laguerre transform, Heat-diffusion, Poisson semigroups, Riesz transform

\section{Introduction}

In his monograph Stein (E. M. Stein, 1970) suggested the study of analogues of the fundamental operators in the classical harmonic analysis as well as in the theory of partial differential equations, such as Riesz transforms, conjugate Poisson integrals, multipliers, fractional integrals, maximal functions, square functions, ..., extensively in the literature in many different aspects and in a context of discrete or continuous expansions with respect to eigenfunctions of self-adjoint and positive differential operators that can be considered in the context of the Laguerre operator $L_{\alpha}$.

The study of these objects in the context of orthogonal expansions was initiated by the fundamental work of Muckenhoupt and Stein (B. Muckenhoupt \& E. M. Stein, 1965), which treated, among other things, one-dimensional ultraspherical expansions. Then Muckenhoupt elaborated the necessary tools and investigated Riesz transforms (or rather conjugate mappings) for Hermite and Laguerre expansions (B. Muckenhoupt, 1969; B. Muckenhoupt, 1970; B. Muckenhoupt, 1969). However, he worked in the one-dimensional setting and used methods which are inapplicable in higher dimensions. In fact, passing with Riesz transforms to higher dimensions turned out not to be as straightforward as one could expect.

The importance of analyzing semigroups of completely positive maps on von Neumann algebras has been impressively demonstrated by the recent work of Popa, Peterson and Ozawa (M. Junge \& T. Mei, 2010) and also occurs in the work of Shlyahktenko/Connes (A. Connes \& Dimitri Shlyakhtenko, 2005) on Betti numbers for von Neumann algebras. A common thread in this analysis is to adapt some differential geometric concepts in the setting of von Neumann algebras. It was discovered by P. A. Meyer that the general theory of semigroups provides an appropriate framework to formulate Riesz transforms which relate the norm of different derivatives in the classical setting.

In this paper we investigate the Riesz transform associated with Laguerre operator on the dual of the Laguerre hypergroup. To achieve our goal we use a procedure that will be described below and that was developed for the first time by Torrea and others in J. J. Betancor, J. C. Fariña, L. Rodriguez-Mesa, A, Sanabria-Garcia and J. L. Torrea (2008).

The organization of the paper is the following. Section 2 contains some basic facts and some representations for the semigroups needed in the sequel about the Laguerre hypergroup. In Section 3 we define the heat-diffusion 
semigroup, the Poisson-Laguerre semigroup and the fractional power based on a Laguerre operator.

Finally, Section 4 is devoted to defining the Riesz potential, the higher order Riesz transform on the dual of the Laguerre hypergroup and to proving the main result of this paper (theorem 1 and theorem 2) where we establish that the higher order Riesz transform is a principal value singular integral operator and it is a multiplier operator under the Fourier transform.

Throughout this paper, $C$ will always represent a positive constant, not necessarily the same in each occurrence.

\section{Preliminaries}

In this section we set some notations and some basic results about the laguerre hypergroup. As basic references of the Laguerre hypergroup, we refer the reader to (M. Assal \& H. Ben Abdallah, 2005; M. M. Nessibi \& K. Trimeche, 1997).

Notations:

We denote by:

- $\mathbb{K}=[0, \infty] \times \mathbb{R}$ equipped with the weighted Lebesgue measure $m_{\alpha}$ on $\mathbb{K}$ given by

$$
d m_{\alpha}(x, t)=\frac{x^{2 \alpha+1} d x d t}{\pi \Gamma(\alpha+1)}, \quad \alpha \geq 0 .
$$

For every $1 \leq p \leq \infty$,we denote by $L^{p}(\mathbb{K})=L^{p}\left(\mathbb{K}, d m_{\alpha}\right)$ the spaces of complex-valued functions $f$, measurable on $\mathbb{K}$ such that:

$$
\|f\|_{L^{p}(\mathbb{K})}=\left(\int_{\mathbb{K}}|f(x, t)|^{p} d m_{\alpha}(x, t)\right)^{\frac{1}{p}}<\infty, \text { if } p \in[1, \infty] .
$$

and

$$
\|f\|_{L^{\infty}(\mathbb{K})}=\operatorname{ess} \sup _{(x, t) \in \mathbb{K}}|f(x, t)| .
$$

- $\mathcal{D}(\mathbb{K})$ the space of $C^{\infty}$ functions and having compact support on $\mathbb{K}$.

- $\hat{\mathbb{K}}=\mathbb{R} \times \mathbb{N}$ the dual space of $\mathbb{K}$.

- $L^{p}(\hat{\mathbb{K}})=L^{p}\left(\hat{\mathbb{K}}, d \gamma_{\alpha}\right)$ the spaces of complex-valued functions $f$, measurable on $\hat{\mathbb{K}}$ such that:

$$
\|f\|_{L^{p}(\hat{\mathbb{K}})}=\left(\int_{\hat{\mathbb{K}}}|f(\lambda, m)|^{p} d \gamma_{\alpha}(\lambda, m)\right)^{\frac{1}{p}}<\infty, \text { if } 1 \leq p<\infty
$$

and

$$
\|f\|_{L^{\infty}(\hat{\mathbb{K}})}=\operatorname{ess} \sup _{(\lambda, m) \in \hat{\mathbb{K}}}|f(\lambda, m)|
$$

where $d \gamma_{\alpha}(\lambda, m)$ being the positive measure defined on $\hat{\mathbb{K}}$ by:

$$
\int_{\hat{\mathbb{K}}} f(\lambda, m) d \gamma_{\alpha}(\lambda, m)=\sum_{m=0}^{\infty} L_{m}^{(\alpha)}(0) \int_{\mathbb{R}} f(\lambda, m)|\lambda|^{\alpha+1} d \lambda,
$$

For $(x, t) \in] 0, \infty[\times \mathbb{R}$ and $\alpha \in[0, \infty]$, we consider the following partial differential operator, named the Laguerre operator:

$$
L=\frac{\partial^{2}}{\partial x^{2}}+\frac{2 \alpha+1}{x} \frac{\partial}{\partial x}+x^{2} \frac{\partial^{2}}{\partial t^{2}}
$$

Remark 1 For $\alpha=n-1, n \in \mathbb{N}^{*}$, the operator $L$ is the radial part of the sublaplacian on the Heisenberg group $\mathbb{H}^{n}$. For $(\lambda, m) \in \hat{\mathbb{K}}$ and $(x, t) \in \mathbb{K}$, we put $\varphi_{\lambda, m}(x, t)=e^{i \lambda t} \mathcal{L}_{m}^{(\alpha)}\left(|\lambda| x^{2}\right)$, where $\mathcal{L}_{m}^{(\alpha)}$ is the Laguerre function defined on $[0, \infty]$ by $\mathcal{L}_{m}^{(\alpha)}(x)=e^{\frac{-x}{2}} L_{m}^{(\alpha)}(x) / L_{m}^{(\alpha)}(0)$ and $L_{m}^{(\alpha)}$ is the Laguerre polynomial of degree $m$ and order $\alpha$.

Proposition 1 For $(\lambda, m) \in \hat{\mathbb{K}}$, the function $\varphi_{\lambda, m}$, is the unique solution of the following problem (N. M. Lebedev, 1972; A, Erdélyi, W. Magnus, F. Aberthettinger, \& G. Tricomi, 1993):

$$
\left\{\begin{array}{c}
L u=-\mathcal{N}(\lambda, m) u \\
u(0,0)=1 \\
\partial_{x} u(0, t)=0, \forall t \in \mathbb{R}
\end{array}\right.
$$


Where

$$
\mathcal{N}(\lambda, m)=4|\lambda|\left(m+\frac{\alpha+1}{2}\right)
$$

We recall for $(\lambda, m) \in \hat{\mathbb{K}}$ and for a suitable function $f: \mathbb{K} \longrightarrow \mathbb{C}$, the Fourier-Laguerre transform is defined in (M. M. Nessibi \& K. Trimeche, 1997; M. Assal \& H. Ben Abdallah, 2005) by:

$$
F(f)(\lambda, m)=\int_{\mathbb{K}} f(x, t) \varphi_{-\lambda, m}(x, t) d m_{\alpha}(x, t), \quad(\lambda, m) \in \hat{\mathbb{K}}
$$

It has been proved in M. M. Nessibi \& K. Trimeche (1997) that the Fourier-Laguerre transform given above is a topological isomorphism from $S(\mathbb{K})$ to $S(\hat{\mathbb{K}})$ and its inverse is given by

$$
G(f)(x, t)=\int_{\hat{\mathbb{K}}} f(\lambda, m) \varphi_{\lambda, m}(x, t) d \gamma_{\alpha}(\lambda, m), \quad(x, t) \in \mathbb{K}
$$

where

- $S(\mathbb{K})$ is the Schwartz space of functions $\psi: \mathbb{R}^{2} \longrightarrow \mathbb{C}$ even with respect to the first variable, $C^{\infty}$ on $\mathbb{R}^{2}$ and rapidly decreasing together with all their derivatives; i.e. for all $k, p, q \in \mathbb{N}$, we have

$$
\widetilde{\mathcal{N}}_{k, p, q}(\psi)=\sup _{(x, t) \in \mathbb{K}}\left\{\left(1+x^{2}+t^{2}\right)^{k}\left|\frac{\partial^{p+q}}{\partial x^{p} \partial t^{q}} \psi(x, t)\right|\right\}<\infty .
$$

- $S(\mathbb{K})$ the space of functions $\Psi: \hat{\mathbb{K}} \longrightarrow \mathbb{C}$ satisfying:

i) For all $m, p, q, r, s \in \mathbb{N}$ the function

$$
\lambda \longrightarrow \lambda^{p}\left(|\lambda|\left(m+\frac{\alpha+1}{2}\right)\right)^{q} \Lambda_{1}^{r}\left(\Lambda_{2}+\frac{\partial}{\partial \lambda}\right)^{s} \Psi(\lambda, m)
$$

is bounded and continuous on $\mathbb{R}, C^{\infty}$ on $\mathbb{R}^{*}=\mathbb{R} \backslash 0$ and such that the left and the right derivatives at zero exist.

ii) For all $k, p, q \in \mathbb{N}$, we have

$$
v_{k, p, q}(\Psi)=\sup _{(\lambda, m) \in \mathbb{R}^{*} \times \mathbb{N}}\left\{\left(1+\lambda^{2}\left(1+m^{2}\right)\right)^{k}\left|\Lambda_{1}^{p}\left(\Lambda_{2}+\frac{\partial}{\partial \lambda}\right)^{q} \Psi(\lambda, m)\right|\right\}<\infty .
$$

where

- $\Lambda_{1} \Psi(\lambda, m)=\frac{1}{|\lambda|}\left(m \Delta_{+} \Delta_{-} \Psi(\lambda, m)+(\alpha+1) \Delta_{+} \Psi(\lambda, m)\right)$.

- $\Lambda_{2} \Psi(\lambda, m)=-\frac{1}{2 \lambda}\left((\alpha+m+1) \Delta_{+} \Psi(\lambda, m)+m \Delta_{-} \Psi(\lambda, m)\right)$.

- $\Delta_{+} \Psi(\lambda, m)=\Psi(\lambda, m+1)-\Psi(\lambda, m)$.

- $\Delta_{-} \Psi(\lambda, m)=\left\{\begin{array}{cc}\Psi(\lambda, m)-\Psi(\lambda, m-1), & \text { if } \quad m \geq 1 \\ \Psi(\lambda, 0)=1, & \text { if } m=0 .\end{array}\right.$

We note that $S(\mathbb{K})$ (resp. $S(\hat{\mathbb{K}})$ ) equipped with the semi-norms $\widetilde{\mathcal{N}}_{k, p, q}\left(\right.$ resp. $\left.v_{k, p, q}\right)$ is a Fréchet space (M. M. Nessibi \& K. Trimeche, 1997).

Proposition 2 For all $(\lambda, m) \in \mathbb{R}^{*} \times \mathbb{N}$ and $(x, t) \in \mathbb{K}$, we have

$$
\begin{gathered}
\Lambda_{1} \varphi_{\lambda, m}(x, t)=-x^{2} \varphi_{\lambda, m}(x, t), \\
\left(\Lambda_{2}+\frac{\partial}{\partial \lambda}\right) \varphi_{\lambda, m}(x, t)=i t \varphi_{\lambda, m}(x, t) .
\end{gathered}
$$

Proof. For all $m \geq 1$, we have in M. M. Nessibi \& K. Trimeche (1997)

$$
\begin{gathered}
x L_{m}^{(\alpha)}(x)=(2 m+\alpha+1) L_{m}^{(\alpha)}(x)-(m+1) L_{m+1}^{(\alpha)}(x)-(m+\alpha) L_{m-1}^{(\alpha)}(x), \\
x \frac{d}{d x} L_{m}^{(\alpha)}(x)=m L_{m}^{(\alpha)}(x)-(m+\alpha) L_{m-1}^{(\alpha)}(x) .
\end{gathered}
$$


We deduce the results from these identities.

Using these operators given in a above proposition and as in Miloud Assal (2009), we define operator on $\hat{\mathbb{K}}$

$$
\mathcal{L}=\Lambda_{1}^{2}-\left(\Lambda_{2}+\frac{\partial}{\partial \lambda}\right)^{2}
$$

it is easily to see that

$$
\mathcal{L} \varphi_{\lambda, m}(x, t)=N(x, t) \varphi_{\lambda, m}(x, t)
$$

Where the topology on $\mathbb{K}$ is given by the norm $N(x, t)=x^{4}+t^{2}$, while we assign to $\hat{\mathbb{K}}$ the topology generated by the quasi-semi-norm $\mathcal{N}(\lambda, m)=4|\lambda|\left(\alpha+\frac{m+1}{2}\right)$. In fact, the dual of the Laguerre hypergroup $\hat{\mathbb{K}}$ can be topologically identified with the so-called Heisenberg fan , i.e., the subset embedded in $R^{2}$ given by (Miloud Assal, 2009)

$$
\left(\bigcup_{m \in \mathbb{N}}\left\{(\lambda, \mu) \in \mathbb{R}^{2}: \mu=|\lambda|(2 m+\alpha+1), \lambda \neq 0\right\}\right) \bigcup\left\{(0, \mu) \in \mathbb{R}^{2}: \mu \geq 0\right\} .
$$

Moreover, the subset $\left\{(0, \mu) \in \mathbb{R}^{2}: \mu \geq 0\right\}$ has zero Plancherel measure, therefore it will usually be disregarded.

It is easily to see that for an appropriate function $\Phi$ it follows that

$$
G[(1+\mathcal{L}) \Phi](x, t)=[1+N(x, t)] G(\Phi)(x, t)
$$

We recall that $(\mathbb{K}, *, i)$ is an hypergroup in the sense of Jewett (R. I. Jewett, 1975) where $i$ denotes the involution defined on $\mathbb{K}$ by $i(x, t)=(x,-t)$. This hypergroup is the Laguerre hypergroup which can been seen as a deformation of the hypergroup of radial functions on the Heisenberg group (M. Assal \& H. Ben Abdallah, 2005).

For $(x, t) \in \mathbb{K}$ and $(\lambda, m) \in \hat{\mathbb{K}}$, Let

$$
P_{(\lambda, m)} f(x, t)=G(f)(x, t) \varphi_{-\lambda, m}(x, t) .
$$

Definition 1 The generalized translation operators $T_{(x, t)}^{(\alpha)}$ on the Laguerre hypergroup are given for a suitable function $f$ by:

$$
T_{(x, t)}^{(\alpha)} f(y, s)=\left\{\begin{array}{c}
\frac{1}{2 \pi} \int_{0}^{2 \pi} f\left(((x, t),(y, s))_{\theta, 1}\right) d \theta, \text { if } \alpha=0 \\
\frac{\alpha}{\pi} \int_{0}^{1}\left(\int_{0}^{2 \pi} f\left(((x, t),(y, s))_{\theta, r}\right) d \theta\right) r\left(1-r^{2}\right)^{\alpha-1} d r, \quad \text { if } \alpha>0
\end{array}\right.
$$

They satisfy the following properties (see M. Assal \& V. S. Guliyev, 2006):

- $T_{(0,0)}^{(\alpha)} f(y, s)=f(y, s)$,

- $T_{(x, t)}^{(\alpha)} f(y, s)=T_{(y, s)}^{\alpha} f(x, t)$,

- $T_{(x, t)}^{(\alpha)} \varphi_{\lambda, m}(y, s)=\varphi_{\lambda, m}(x, t) \varphi_{\lambda, m}(y, s)$, for $(\lambda, m) \in \hat{\mathbb{K}}$

- $F\left(T_{(x, t)}^{(\alpha)} f\right)(\lambda, m)=F(f)(\lambda, m) \varphi_{\lambda, m}(x, t)$, for $(\lambda, m) \in \hat{\mathbb{K}}$,

- $\left\|T_{(x, t)}^{(\alpha)} f\right\|_{L^{p}(\mathbb{K})} \leq\|f\|_{L^{p}(\mathbb{K})}$ for $f \in L^{p}(\mathbb{K})$,

- The convolution product of $h$ and $g$ in the space $L^{1}(\mathbb{K})$ is defined by:

$$
(h * g)(x, t)=\int_{\mathbb{K}} T_{(x, t)}^{(\alpha)} h(y, s) g(y,-s) d m_{\alpha}(y, s), \text { for }(x, t) \in \mathbb{K} .
$$

Definition 2 The generalized translation operators $T_{(\lambda, m)}^{(\alpha)}$ on $\hat{\mathbb{K}}$ are given for a suitable function $f$ by:

$$
\left[T_{(\lambda, m)}^{(\alpha)} f\right](\mu, n)=\sum_{j \in \mathbb{N}_{m, n}} f(\lambda+\mu, j) C_{j}^{\alpha}((\lambda, m)(\mu, n))
$$

where

and

$$
C_{j}^{\alpha}((\lambda, m)(\mu, n))=\frac{L_{j}^{\alpha}(0)}{\Gamma(\alpha+1)} \int_{0}^{\infty} \mathcal{L}_{m}^{\alpha}\left(\mid \frac{\lambda}{\lambda+\mu \mid} x\right) \mathcal{L}_{n}^{\alpha}\left(\mid \frac{\mu}{\lambda+\mu \mid} x\right) \mathcal{L}_{j}^{\alpha}(x) x^{\alpha} d x
$$

$$
\mathbb{N}_{m, n}=\left\{\begin{array}{cc}
\{0,1, \ldots, m+n\}, & \text { if } \lambda+\mu \neq 0 \text { and } \lambda \mu>0, \\
\mathbb{N}, & \text { if } \lambda+\mu \neq 0 \text { and } \lambda \mu \leq 0
\end{array}\right.
$$


Remark $2 \forall(\lambda, m),(\mu, n) \in \hat{\mathbb{K}}$, we have (see Miloud Assal, 2009):

$$
\sum_{j \in \mathbb{N}_{m, n}} C_{j}^{\alpha}((\lambda, m)(\mu, n))=1
$$

- The generalized convolution product on $\hat{\mathbb{K}}$ is defined for a suitable pair of functions $f$ and $g$ by:

$$
f * g(\lambda, m)=\int_{\widehat{\mathbb{K}}} T_{(\lambda, m)}^{(\alpha)} f(\mu, n) g(-\mu, n) d \gamma_{\alpha}(\mu, n), \quad \forall(\lambda, m) \in \hat{\mathbb{K}}
$$

Proposition 3 The following properties hold (see Miloud Assal, 2009):

1) For all $(\lambda, m),(\mu, n) \in \hat{\mathbb{K}}$ and for an appropriate function $f$ on $\hat{\mathbb{K}}$, we have:

(i) $T_{(0,0)}^{(\alpha)} f(\lambda, m)=f(\lambda, m), \forall(\lambda, m) \in \hat{\mathbb{K}}$

$(i i) T_{(\lambda, m)}^{(\alpha)} f(\mu, n)=T_{(\mu, n)}^{(\alpha)} f(\lambda, m), \forall(\lambda, m),(\mu, n) \in \hat{\mathbb{K}}$.

$(i i i) T_{(\lambda, m)}^{(\alpha)} \varphi_{(\mu, n)}(x, t)=\varphi_{(\lambda, m)}(x, t) \varphi_{(\mu, n)}(x, t), \forall(x, t) \in \mathbb{K}$.

2) Let $f \in L^{p}\left(d \gamma_{\alpha}\right), 1<p<\infty$. Then for all $(\lambda, m) \in \hat{\mathbb{K}}$, the function $T_{(\lambda, m)}^{(\alpha)}$ f belongs to $L^{p}\left(d \gamma_{\alpha}\right)$ and we have:

$$
\left\|T_{(\lambda, m)}^{(\alpha)} f\right\|_{p} \leq\|f\|_{p} .
$$

3) For $f \in L^{p}\left(d \gamma_{\alpha}\right)$ and $g \in L^{p}\left(d \gamma_{\alpha}\right), 1 \leq p, q \leq \infty$ the function $f * g$ belongs to $L^{r}\left(d \gamma_{\alpha}\right), \frac{1}{p}+\frac{1}{q}=1+\frac{1}{r}$, and we have

$$
\|f * g\| \leq\|f\|_{p}\|g\|_{q}
$$

4) Let $f, g \in L^{1}\left(d \gamma_{\alpha}\right)$, then we have

$$
G(f * g)=G(f) G(g) .
$$

5) Let $f \in L^{1}\left(d \gamma_{\alpha}\right)$, then for all $(x, t) \in \mathbb{K}$ and $(\lambda, m) \in \hat{\mathbb{K}}$, we have

$$
G\left(T_{(\lambda, m)}^{(\alpha)} f\right)(x, t)=\varphi_{-\lambda, m}(x, t) G(f)(x, t) .
$$

\section{Semigroups}

\subsection{The Heat-diffusion Semigroup}

The heat-diffusion semigroup $\left\{T_{t}\right\}_{t \geq 0}$, associated to $(\mathcal{L})$, is defined by (A. Pazy, 1983; R. Radha \& D. Venku Naidu, 2008)

$$
\begin{aligned}
T_{t} f(\lambda, m) & :=e^{-t \mathcal{L}} f(\lambda, m) \\
& =\int_{\mathbb{K}} e^{-t N(y, s)} P_{(\lambda, m)} f(y, s) d m_{\alpha}(y, s) \\
& =\int_{\mathbb{K}} e^{-t N(y, s)}\left(\int_{\hat{\mathbb{K}}} f(\mu, n) \varphi_{\mu, n}(y, s) d \gamma_{\alpha}(\mu, n)\right) \varphi_{-\lambda, m}(y, s) d m_{\alpha}(y, s) \\
& =\int_{\hat{\mathbb{K}}} f(\mu, n)\left[\int_{\mathbb{K}} e^{-t N(y, s)} \varphi_{-\lambda, m}(y, s) \varphi_{\mu, n}(y, s) d m_{\alpha}(y, s)\right] d \gamma_{\alpha}(\mu, n) \\
& =\int_{\widehat{\mathbb{K}}} f(\mu, n) T_{t}((\lambda, m),(\mu, n)) d \gamma_{\alpha}(\mu, n)
\end{aligned}
$$

where

$$
\begin{aligned}
T_{t}((\lambda, m),(\mu, n)) & =\int_{\mathbb{K}} e^{-t N(y, s)} \varphi_{-\lambda, m}(y, s) \varphi_{\mu, n}(y, s) d m_{\alpha}(y, s) \\
& =\int_{\mathbb{K}} e^{-t N(y, s)}\left[T_{(-\lambda, m)}^{(\alpha)} \varphi_{(\mu, n)}(y, s)\right] d m_{\alpha}(y, s)
\end{aligned}
$$

is the heat kernel of the integral representation $T_{t} f$.

Proposition 4 This semigroup $\left\{T_{t}\right\}_{t \geq 0}$ is a strongly continuous semigroup on $L^{p}(\hat{\mathbb{K}})$ with infinitesimal generator L. 
Proof. Let $f \in L^{p}(\hat{\mathbb{K}})$, then

$$
\lim _{s \longrightarrow t}\|T(s) f-T(t) f\|_{L^{p}(\hat{\mathbb{K}})}=\lim _{s \longrightarrow t}\|(T(s)-T(t)) f\|_{L^{p}(\widehat{\mathbb{K}})} \leq \lim _{s \longrightarrow t}\|T(s)-T(t)\|_{L^{p}(\hat{\mathbb{K}})}\|f\|_{L^{p}(\hat{\mathbb{K}})}=0 .
$$

By the definition of the heat-diffusion semigroup $\left\{T_{t}\right\}_{t \geq 0}$, we establish the following property.

Corollary 1 For $(y, s) \in \mathbb{K}$ and $(\lambda, m) \in \hat{\mathbb{K}}$, we have

$$
T_{t} \varphi_{\lambda, m}(y, s)=e^{-t N(y, s)} \varphi_{\lambda, m}(y, s),
$$

Proof. we have

$$
\begin{aligned}
T_{t} \varphi_{\lambda, m}(y, s) & =\int_{\hat{\mathbb{K}}} \int_{\mathbb{K}} e^{-t N(y, s)} \varphi_{\lambda, m}(\mu, n) \varphi_{-\lambda, m}(y, s) \varphi_{\lambda, m}(\mu, n) d m_{\alpha}(u, v) d \gamma_{\alpha}(\mu, n) \\
& =\int_{\mathbb{K}} \varphi_{\mu, \eta}(u, v)\left(\int_{\mathbb{K}} e^{-t c_{\lambda, m}} \varphi_{-\lambda, m}(u, v) \varphi_{\lambda, m}(y, s) d \gamma_{\alpha}(\lambda, m)\right) d m_{\alpha}(u, v) \\
& =\int_{\mathbb{K}} \varphi_{\mu, \eta}(u, v) F^{-1}\left(e^{-t c_{.,}} \varphi_{-\ldots}(u, v)\right)(y, s) d m_{\alpha}(u, v) \\
& =F\left(F^{-1}\left(e^{-t c_{\ldots,}} \varphi_{\ldots, . .}(y, s)\right)\right)(-\mu, \eta) \\
& =e^{-t c_{\mu, \eta}} \varphi_{\mu, \eta}(y, s) .
\end{aligned}
$$

\subsection{The Fractional Power}

For $\delta>0$, the negative power $\mathcal{L}^{-\delta}$ of $\mathcal{L}$ with respect to the measure $d \gamma_{\alpha}$ is defined, as in (K. Stempak \& Jose Luis Torrea, 2003), by

$$
\mathcal{L}^{-\delta} f(\lambda, m):=\int_{\mathbb{K}} \frac{P_{(\lambda, m)} f(y, s)}{N(y, s)^{\delta}} \varphi_{\lambda, m}(y, s) d m_{\alpha}(y, s), \quad f \in L^{2}\left(\hat{\mathbb{K}}, d \gamma_{\alpha}\right)
$$

It is not hard to prove that $\mathcal{L}^{-\delta}$ can be expressed, for $f \in L^{2}\left(\hat{\mathbb{K}}, d \gamma_{\alpha}\right)$, by means of the following integral

$$
\mathcal{L}^{-\delta} f(\lambda, m)=\frac{1}{\Gamma(\delta)} \int_{0}^{\infty} t^{\delta-1} T_{t} f(\lambda, m) d t,
$$

$\mathcal{L}^{-\delta}$ is also called $\delta$-th fractional integral associated with $\mathcal{L}$. This kind of fractional integrals has been investigated by several authors (G. Gasper, K. Stempak, \& W. Trebels, 1995; P. Graczyk, J. L. Loeb, I. A. Lopez, A. Nowak, \& W. Urbina, 2005; Y. Kanjin \& E. Sato, 1995; K. Stempak,1994).

Corollary 2 If $f(y, s)=\varphi_{\lambda, m}(y, s)$, we have

$$
\mathcal{L}^{-\delta} \varphi_{\lambda, m}(y, s)=\frac{1}{N(y, s)^{\delta}} \varphi_{\lambda, m}(y, s),
$$

Proof. the proof is trivially by using $\Gamma(\delta)=\int_{0}^{\infty} t^{\delta-1} e^{-t} d t$ and the change of variable $u=t \sqrt{N(y, s)}$.

\subsection{The Poisson-Laguerre Semigroup}

The Poisson-Laguerre semigroup $\left\{P_{t}\right\}_{t \geq 0}$, associated to $(\mathcal{L})$, is given by

$$
\begin{aligned}
P_{t} f(\lambda, m) & :=e^{-t \mathcal{L}^{1 / 2}} f(\lambda, m) \\
& =\int_{\mathbb{K}} e^{-t \sqrt{N(y, s)}}\left[P_{(\lambda, m)} f\right](y, s) d m_{\alpha}(y, s) .
\end{aligned}
$$

Now,by using the Bochner subordination formula

$$
e^{-\beta}=\frac{\beta}{\sqrt{4 \pi}} \int_{0}^{\infty} s^{-3 / 2} e^{-s} e^{-\beta^{2} / 4 s} d s
$$

we obtain, after the change of variable $w=\frac{t^{2} N(y, s)}{4 s}$,

$$
P_{t} f(\lambda, m)=\frac{1}{\sqrt{\pi}} \int_{0}^{\infty} \frac{e^{-w}}{\sqrt{w}} T_{\frac{t^{2}}{4 w}} f(\lambda, m) d w .
$$


Proposition 5 This semigroup $\left\{P_{t}\right\}_{t \geq 0}$ is also a strongly continuous semigroup on $L^{p}(\mathbb{K})$, with infinitesimal generator $L^{1 / 2}$.

Proof. we use the fact that $T_{\frac{t^{2}}{4 w}}$ is strongly continuous.

By the definition of the Poisson-Laguerre semigroup $\left\{P_{t}\right\}_{t \geq 0}$, we establish also the following property

Corollary 3 For $(\mu, \eta) \in \hat{\mathbb{K}}$, we have

$$
P_{t} \varphi_{\mu, \eta}(y, s)=e^{-t \sqrt{c_{\mu, \eta}}} \varphi_{\mu, \eta}(y, s)
$$

Proof. We replace $N(y, s)$ by $\sqrt{N(y, s)}$ in the proof of corollary 1 , then the result is immediate.

\section{Higher Order Riesz Transform}

\subsection{The Riesz Potential}

For $\delta>0$, the Riesz potential of order $\delta, I_{\delta}$, with respect to the measure $d \gamma_{\alpha}$ is defined, as in the classical case (Iris A. Lopez \& Wilfredo O. Urbina, 2004), by

$$
I_{\delta}:=(-\mathcal{L})^{-\delta / 2}
$$

Proposition 6 The Riesz potential can be also writed as

$$
I_{\delta} f(\lambda, m)=\frac{1}{\Gamma(\delta)} \int_{0}^{\infty} t^{\delta-1} P_{t} f(\lambda, m) d t
$$

Proof. By using $(-\mathcal{L})^{-\delta}$, we have

$$
I_{\delta} f(\lambda, m)=\frac{1}{\Gamma(\delta / 2)} \int_{0}^{\infty} t^{\delta / 2-1} T_{t} f(\lambda, m) d t
$$

After to replace $P_{t} f(\lambda, m)$ with his expression, the change of variable $t^{\prime}=\frac{t^{2}}{4 u}$ and the property of the function Gamma, we obtain:

$$
\frac{1}{\Gamma(\delta / 2)} \int_{0}^{\infty} t^{\delta / 2-1} T_{t} f(\lambda, m) d t-\frac{1}{\Gamma(\delta)} \int_{0}^{\infty} t^{\delta-1} P_{t} f(\lambda, m) d t=0
$$

Corollary 4 If $f(y, s)=\varphi_{\lambda, m}(y, s)$, we have

$$
I_{\delta} \varphi_{\lambda, m}(y, s)=\frac{1}{N(y, s)^{\delta / 2}} \varphi_{\lambda, m}(y, s)
$$

Proof. the proof is trivially by using $\Gamma(\delta)=\int_{0}^{\infty} t^{\delta-1} e^{-t} d t$ and the change of variable $u=t \sqrt{N(y, s)}$.

4.2 The Riesz Transform

For $\lambda \in \mathbb{R}^{*}$, the operator given by (2) can be written in the form:

$$
\begin{aligned}
\mathcal{L} & =\frac{1}{2}\left\{\left[\Lambda_{1}-\left(\Lambda_{2}+\frac{\partial}{\partial \lambda}\right)\right]\left[\Lambda_{1}+\left(\Lambda_{2}+\frac{\partial}{\partial \lambda}\right)\right]+\left[\Lambda_{1}+\left(\Lambda_{2}+\frac{\partial}{\partial \lambda}\right)\right]\left[\Lambda_{1}-\left(\Lambda_{2}+\frac{\partial}{\partial \lambda}\right)\right]\right\} \\
& =\frac{1}{2}\left\{\Lambda \Lambda^{*}+\Lambda^{*} \Lambda\right\}
\end{aligned}
$$

where $\Lambda=\Lambda_{1}-\left(\Lambda_{2}+\frac{\partial}{\partial \lambda}\right)$ and $\Lambda^{*}=\Lambda_{1}+\left(\Lambda_{2}+\frac{\partial}{\partial \lambda}\right)$ are formal adjoint operators in $L^{2}\left(\hat{\mathbb{K}}, d \gamma_{\alpha}\right)$.

Then the factorization of $\mathcal{L}$ suggests to define (formally), as in (K. Stempak \& J. L. Torrea, 2003) the Riesz transform $R_{\alpha}$ associated with $\mathcal{L}$ by

$$
\begin{aligned}
R & :=\Lambda \mathcal{L}^{-\frac{1}{2}} \\
& =\left[\Lambda_{1}-\left(\Lambda_{2}+\frac{\partial}{\partial \lambda}\right)\right] \mathcal{L}^{-\frac{1}{2}}
\end{aligned}
$$


Using the definition of the negative power of $\mathcal{L}$ and for a smooth function $f$,we obtain

$$
\begin{aligned}
R f(\lambda, m) & =\Lambda\left[\frac{1}{\Gamma\left(\frac{1}{2}\right)} \int_{0}^{\infty} t^{\frac{-1}{2}} T_{t} f(\lambda, m) d t\right] \\
& =\frac{1}{\Gamma\left(\frac{1}{2}\right)} \int_{0}^{\infty} t^{\frac{-1}{2}} \Lambda T_{t} f(\lambda, m) d t \\
& =\frac{1}{\Gamma\left(\frac{1}{2}\right)} \int_{0}^{\infty} t^{\frac{-1}{2}} \Lambda\left[\int_{\mathbb{K}} f(\mu, n) T_{t}((\lambda, m),(\mu, n)) d \gamma_{\alpha}(\mu, n)\right] d t \\
& =\frac{1}{\Gamma\left(\frac{1}{2}\right)} \int_{0}^{\infty} t^{\frac{-1}{2}}\left[\int_{\mathbb{K}} f(\mu, n) \Lambda T_{t}((\lambda, m),(\mu, n)) d \gamma_{\alpha}(\mu, n)\right] d t \\
& =\int_{\mathbb{K}}\left[\frac{1}{\Gamma\left(\frac{1}{2}\right)} \int_{0}^{\infty} t^{\frac{-1}{2}} \Lambda T_{t}((\lambda, m),(\mu, n)) d t\right] f(\mu, n) d \gamma_{\alpha}(\mu, n) \\
& =\int_{\mathbb{K}} R((\lambda, m),(\mu, n)) f(\mu, n) d \gamma_{\alpha}(\mu, n)
\end{aligned}
$$

where

$$
\begin{aligned}
R((\lambda, m),(\mu, n)) & =\frac{1}{\Gamma\left(\frac{1}{2}\right)} \int_{0}^{\infty} t^{\frac{-1}{2}} \Lambda T_{t}((\lambda, m),(\mu, n)) d t \\
& =\frac{1}{\Gamma\left(\frac{1}{2}\right)} \int_{0}^{\infty} t^{\frac{-1}{2}}\left\{\int_{\mathbb{K}} e^{-t N(y, s)} \Lambda\left[T_{(-\lambda, m)}^{(\alpha)} \varphi_{(\mu, n)}(y, s)\right] d m_{\alpha}(y, s)\right\} d t
\end{aligned}
$$

is the kernel of the integral representation $R f$.

Corollary $5 \operatorname{For}(y, s) \in \mathbb{K}, \lambda, m) \in \hat{\mathbb{K}}$, we have

$$
R \varphi_{\lambda, m}(y, s)=-\frac{y^{2}+i s}{N(y, s)^{1 / 2}} \varphi_{\lambda, m}(y, s)
$$

Proof. Using the proposition 2 and the definition of the Riesz potential $\mathcal{L}^{-1 / 2}$.

Now, we shall prove that the Riesz transform $R$ associated with the Laguerre operator on the dual of the Laguerre hypergroup is a principal value singular integral operator.

Theorem 1 For $(\lambda, m) \in \hat{\mathbb{K}}$, the Riesz, transform $R$ can be defining it by

$$
R f(\lambda, m)=\lim _{\epsilon \rightarrow 0^{+}} \int_{\mathbb{K}, N(y, s)>\epsilon}\left\{\int_{\widehat{\mathbb{K}}} \frac{\left(-y^{2}+i s\right) T_{(-\lambda, m)}^{(\alpha)} \varphi_{(\mu, n)}(y, s)}{N(y, s)^{1 / 2}} f(\mu, n) d \gamma_{\alpha}(\mu, n)\right\} d m_{\alpha}(y, s) .
$$

Proof. We have

$$
R f(\lambda, m)=\frac{1}{\Gamma\left(\frac{1}{2}\right)} \int_{\mathbb{K}} \int_{\mathbb{K}} \int_{0}^{\infty} t^{\frac{-1}{2}} e^{-t N(y, s)} \Lambda\left[T_{(-\lambda, m)}^{(\alpha)} \varphi_{(\mu, n)}(y, s)\right] f(\mu, n) d \gamma_{\alpha}(\mu, n) d t d m_{\alpha}(y, s)
$$

Using a change of variable $t^{\prime}=-t N(y, s)$, the function $\Gamma\left(\frac{1}{2}\right)$ and $\Lambda \varphi_{-\lambda, m}(y, s)=\left(-y^{2}+i s\right) \varphi_{\lambda, m}(y, s)$, we obtain that

$$
R f(\lambda, m)=\lim _{\epsilon \rightarrow 0^{+}} \int_{\mathbb{K}, N(y, s)>\epsilon}\left\{\int_{\widehat{\mathbb{K}}} \frac{\left(-y^{2}+i s\right) T_{(-\lambda, m)}^{(\alpha)} \varphi_{(\mu, n)}(y, s)}{N(y, s)^{1 / 2}} f(\mu, n) d \gamma_{\alpha}(\mu, n)\right\} d m_{\alpha}(y, s) .
$$

We want to show that $R f$ is a multiplier operator under the inverse Fourier transform. A linear operator $R f$ is a multiplier operator if $G(R f)(y, s)=m(y, s) G(f)(y, s)$ in the sense that

$$
\begin{aligned}
R f(\lambda, m) & \left.=\int_{\mathbb{K}} m(x, t) G(f)(x, t) \varphi_{-\lambda, m}(x, t)\right) d m_{\alpha}(x, t) \\
& =F[m(., .) G(f)](\lambda, m) .
\end{aligned}
$$


Theorem 2 For $(y, s) \in \mathbb{K}$, we have

$$
G(R f)(y, s)=m(y, s) G(f)(y, s)
$$

and for $f \in L^{p}(\hat{\mathbb{K}}), 1<p<\infty$, we have

$$
\|R f\|_{L^{p}(\hat{\mathbb{K}})} \leq C\|f\|_{L^{p}(\hat{\mathbb{K}})}
$$

Proof.

$$
\begin{aligned}
R f(\lambda, m) & =\lim _{\epsilon \rightarrow 0^{+}} \int_{\mathbb{K}, N(y, s)>\epsilon}\left\{\int_{\mathbb{K}} \frac{\left(-y^{2}+i s\right) T_{(-\lambda, m)}^{(\alpha)} \varphi_{(\mu, n)}(y, s)}{N(y, s)^{1 / 2}} f(\mu, n) d \gamma_{\alpha}(\mu, n)\right\} d m_{\alpha}(y, s) \\
& \lim _{\epsilon \rightarrow 0^{+}} \int_{\mathbb{K}, N(y, s)>\epsilon} \frac{\left(-y^{2}+i s\right)}{N(y, s)^{1 / 2}} \varphi_{(-\lambda, m)}(y, s)\left[\int_{\mathbb{K}} \varphi_{(\mu, n)}(y, s) f(\mu, n) d \gamma_{\alpha}(\mu, n)\right] d m_{\alpha}(y, s) \\
& =\int_{\mathbb{K}} m(y, s) \varphi_{(-\lambda, m)}(y, s) G(f) d m_{\alpha}(y, s) \\
& =F[m(., .) G(f)](\lambda, m)
\end{aligned}
$$

where

$$
m(y, s)=\frac{-y^{2}+i s}{N(y, s)^{1 / 2}} .
$$

Then the result follows by applying the inverse Fourier transform $G$.

For the $L^{p}$-boundedness properties of the Riesz transform, we have:

$$
\begin{aligned}
\|R f\|_{L^{p}(\mathbb{\mathbb { K }})} & =\|F[m(., .) G(f)](\lambda, m)\|_{L^{p}(\mathbb{\mathbb { K }})} \\
& \leq\|[m(., .) G(f)](\lambda, m)\|_{L^{p}(\mathbb{K})} \\
& \leq\|G(f)(\lambda, m)\|_{L^{p}(\mathbb{K})} \\
& \leq C\|f\|_{L^{p}(\hat{\mathbb{K}})}
\end{aligned}
$$

\subsection{The Higher Order Riesz Transform}

For every $k \in \mathbb{N}$, the higher order Riesz transform $R^{(k)}$, associated with $\mathcal{L}$ is defined by

$$
\begin{aligned}
R^{(k)} & :=\Lambda^{k} \mathcal{L}^{-\frac{k}{2}} \\
& =\left[\Lambda_{1}-\left(\Lambda_{2}+\frac{\partial}{\partial \lambda}\right)\right]^{k} \mathcal{L}^{-\frac{k}{2}}
\end{aligned}
$$

Again it is easily to see that

$$
R^{(k)} \varphi_{\lambda, m}(y, s)=(-1)^{k} \frac{\left(x^{2}+i t\right)^{k}}{N(y, s)^{k / 2}} \varphi_{\lambda, m}(y, s)
$$

Proposition 7 For $k \in \mathbb{N}$ and $(\lambda, m) \in \hat{\mathbb{K}}$, we have

$$
R^{(k)} f(\lambda, m)=\lim _{\epsilon \rightarrow 0^{+}} \int_{\mathbb{K}, N(y, s)>\epsilon}\left\{\int_{\hat{\mathbb{K}}} \frac{\left(-y^{2}+i s\right)^{k}\left[T_{(-\lambda, m)}^{(\alpha)} \varphi_{(\mu, n)}(y, s)\right]}{N(y, s)^{k / 2}} f(\mu, n) d \gamma_{\alpha}(\mu, n)\right\} d m_{\alpha}(y, s)
$$

and for $f \in L^{p}(\hat{\mathbb{K}}), 1<p<\infty$, we have

$$
\left\|R^{(k)} f\right\|_{L^{p}(\hat{\mathbb{K}})} \leq C\|f\|_{L^{p}(\hat{\mathbb{K}})}
$$

Proof. We can deduce from theorem 1 that:

$$
\begin{aligned}
R^{(k)} f(\lambda, m) & =R \circ R \circ \ldots \circ R f(\lambda, m) \\
& =R \circ R \circ \ldots R\left[\lim _{\epsilon \rightarrow 0^{+}} \int_{\mathbb{K}, N(y, s)>\epsilon}\left\{\int_{\mathbb{K}} \frac{\left(-y^{2}+i s\right) T_{(-\lambda, m)}^{(\alpha)} \varphi(\mu, n)(y, s)}{N(y, s)^{1 / 2}} f(\mu, n) d \gamma_{\alpha}(\mu, n)\right\} d m_{\alpha}(y, s)\right] \\
& =\lim _{\epsilon \rightarrow 0^{+}} \int_{\mathbb{K}, N(y, s)>\epsilon}\left\{\int_{\mathbb{K}} \frac{\left(-y^{2}+i s\right)^{k}\left[T_{(-\lambda, m)}^{(\alpha)} \varphi_{(\mu, n)}(y, s)\right]}{N(y, s)^{k / 2}} f(\mu, n) d \gamma_{\alpha}(\mu, n)\right\} d m_{\alpha}(y, s) .
\end{aligned}
$$


From theorem 2, we have

$$
\begin{aligned}
\left\|R^{(k)} f\right\|_{L^{p}(\hat{\mathbb{K}})} & =\left\|F\left[m(., .)^{k} G(f)\right](\lambda, m)\right\|_{L^{p}(\hat{\mathbb{K}})} \\
& \leq\left\|\left[m(., .)^{k} G(f)\right](\lambda, m)\right\|_{L^{p}(\mathbb{K})} \\
& \leq\|G(f)(\lambda, m)\|_{L^{p}(\mathbb{K})} \\
& \leq C\|f\|_{L^{p}(\hat{\mathbb{K}})}
\end{aligned}
$$

\section{Acknowledgment}

The authors would like to thank the reviewers for their valuable comments and suggestions.

\section{References}

A. Connes, \& Dimitri Shlyakhtenko. (2005). L $L^{2}$-homology for von Neumann algebras. J. Reine Angew. Math., 586,125-168. http://dx.doi.org/10.1515/crll.2005.2005.586.125

A. Erdélyi, W. Magnus, F. Aberthettinger, \& G. Tricomi. (1993). Higher Transcendental Fonctions. Vol II. New York: M. G. Graw-Hill.

A. Pazy. (1983). Semigroups of Linear Operators and applications to partial differential equations. New York: Springer-Verlag. http://dx.doi.org/10.1007/978-1-4612-5561-1

B. Muckenhoupt. (1969a). Hermite conjugate expansions. Trans. Amer. Math. Soc., 139, $243-260$. http://dx.doi.org/10.1090/S0002-9947-1969-0249918-0

B. Muckenhoupt. (1969b). Poisson integrals for Hermite and Laguerre expansions. Trans. Amer. Math. Soc., 139, 231-242. http://dx.doi.org/10.1090/S0002-9947-1969-0249917-9

B. Muckenhoupt. (1970). Conjugate functions for Laguerre expansions. Trans. Amer. Math. Soc., 147, $403-418$. http://dx.doi.org/10.1090/S0002-9947-1970-0252945-9

B. Muckenhoupt, \& E. M. Stein. (1965). Classical expansions and their relation to conjugate harmonic functions. Trans. Amer. Math. Soc., 118, 17-92. http://dx.doi.org/10.1090/S0002-9947-1965-0199636-9

E. M. Stein. (1970). Topics in Harmonic Analysis related to the Littlewood-Paley theory. Ann. of Math. Studies, 63. Princenton: Princenton Univ. Press.

G. Gasper, K. Stempak, \& W. Trebels. (1995). Fractional integration for Laguerre expansions. Methods Appl. Anal., 2, 67-75.

Iris A. Lopez, \& Wilfredo O. Urbina. (2004). Fractional differentiation for the Gaussian measure and applications. Bulletin des sciences mathematiques, 128, 587-603. http://dx.doi.org/10.1016/j.bulsci.2004.03.009

J. J. Betancor, J. C. Fariña, L. Rodriguez-Mesa, A. Sanabria-Garcia, \& J. L. Torrea. (2008). Transference between Laguerre and Hermite settings. J. Funct. Anal., 254, 826-850. http://dx.doi.org/10.1016/j.jfa.2007.10.014

K. Stempak. (1994). Heat-diffusion and Poisson integrals for Laguerre expansions. Tohoku Math. J., 2(46), 83-104. http://dx.doi.org/10.2748/tmj/1178225803

K. Stempak, \& Jose Luis Torrea. (2003). Poisson integrals and Riesz transforms for Hermite function expansions with weights. Journal of functional analysis, 202, 443-472. http://dx.doi.org/10.1016/S0022-1236(03)00083-1

M. Assal, \& H. Ben Abdallah. (2005). Generalized Besov type spaces on the Laguerre hypergroup. Annales Mathematiques Blaise Pascal, 12, 117-145. http://dx.doi.org/10.5802/ambp.198

M. Assal, \& V. S. Guliyev. (2006). On maximal function on the Laguerre Hypergroup. Fractional Calculus and Applied Analysis, 9(3), 307-318.

Miloud Assal. (2009). Generalized Besov type spaces on the dual of the Laguerre hupergroup. Acta Mathematica Sinica, English Series, 25(3).

Miloud Assal. (2009). Pseudo-differential operators associated with Laguerre hypergroups. Journal of Computational and Applied Mathematics, 233, (3), 617-620. http://dx.doi.org/10.1016/j.cam.2009.02.028

M. Junge, \& T. Mei. (2010). Noncommutative Riesz transforms-a probabilistic approach. American journal of mathematics, 132(3), 611-680. http://dx.doi.org/10.1353/ajm.0.0122 
M. M. Nessibi, \& K. Trimeche. (1997). Inversion of the Radon transform on the Laguerre Hypergroup by using Generalized Wavelets. Journal of Mathematical Analysis and Applications, $208,337-363$. http://dx.doi.org/10.1006/jmaa.1997.5299

N. M. Lebedev. (1972). Special fonction and their applications. New York: Dover.

P. Graczyk, J. L. Loeb, I. A. Lopez, A. Nowak, \& W. Urbina. (2005). Higher order Riesz transforms, fractional derivatives and Sobolev spaces for Laguerre expansions. J. Math. Pures Appl., 9(84), 375-405. http://dx.doi.org/10.1016/j.matpur.2004.09.003

R. I. Jewett. (1975). spaces with an abstract convolution of measures. Adv. in Math., 18, 1-101. http://dx.doi.org/10.1016/0001-8708(75)90002-X

R. Radha, \& D. Venku Naidu. (2008). Image of $L_{p}\left(\mathbb{R}^{n}\right)$ under the Hermite Semigroup. Hindawi Publishing Corporation International Journal of Mathematics and Mathematical Sciences. http://dx.doi.org/10.1155/2008/287218

S. Thangavelu, \& Yuan Xu. (2007). Riesz transform and Riesz potentials for Dunkl transform. Journal of Computational and Applied Mathematics, 199(1), 181-195. http://dx.doi.org/10.1016/j.cam.2005.02.022

Y. Kanjin, \& E. Sato. (1995). The Hardy-Littlewood theorem on fractional integration for Laguerre series. Proc. Amer. Math. Soc., 123, 2165-2171. 